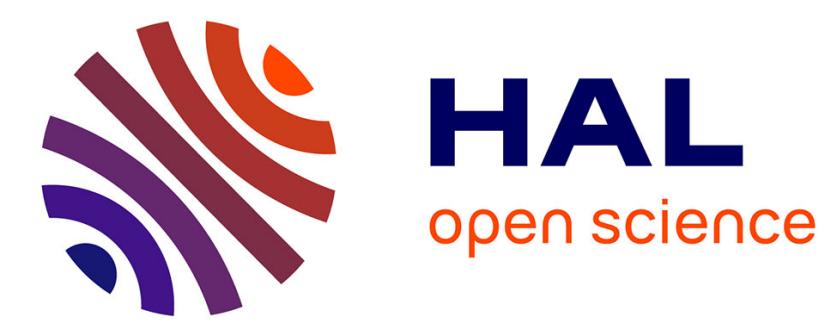

\title{
A Petri Net Model of an Integrated System for the Health Care at Home Management
}

Maria Pia Fanti, Agostino Marcello Mangini, Walter Ukovich, Jean-Jacques

Lesage, Kevin Viard

\section{- To cite this version:}

Maria Pia Fanti, Agostino Marcello Mangini, Walter Ukovich, Jean-Jacques Lesage, Kevin Viard. A Petri Net Model of an Integrated System for the Health Care at Home Management. 2014 IEEE International Conference on Automation Science and Engineering (CASE), Aug 2014, Taipei, Taiwan. pp. 582-587. hal-01061270

\section{HAL Id: hal-01061270 https://hal.science/hal-01061270}

Submitted on 11 Sep 2014

HAL is a multi-disciplinary open access archive for the deposit and dissemination of scientific research documents, whether they are published or not. The documents may come from teaching and research institutions in France or abroad, or from public or private research centers.
L'archive ouverte pluridisciplinaire HAL, est destinée au dépôt et à la diffusion de documents scientifiques de niveau recherche, publiés ou non, émanant des établissements d'enseignement et de recherche français ou étrangers, des laboratoires publics ou privés. 


\title{
A Petri Net Model of an Integrated System for the Health Care at Home Management
}

\author{
Maria Pia Fanti, Senior Member, IEEE ${ }^{1}$, Agostino Marcello Mangini ${ }^{2}$, \\ Walter Ukovic, Member, IEEE ${ }^{3}$, Jean-Jacques Lesage, Member, IEEE ${ }^{4}$ and Kevin Viard ${ }^{5}$
}

\begin{abstract}
The Health At Home (HAH) is an alternative to the traditional hospital to promote the early discharge and to help patients and elderly people to live autonomously. This paper specifies and models an Integrated System (IS) devoted to the HAH management at the operational level. The IS is designed to monitor the daily living of the apartment inhabitant, detect the possible troubles and accidents, communicate with family, doctors and emergency services. A Petri net model in a modular approach is proposed, in order to effectively describe the actions and the activities of the IS.
\end{abstract}

\section{INTRODUCTION}

The people life expectancy is projected to increase in the next decades. Hence, it is necessary to improve the autonomy and the independence of old or disabled people: Ambient Assisted Living (AAL) technologies and Healthcare at Home $(H A H)$ are new alternatives to the hospitals to promote secure and autonomous life to weak people. In particular, the HAH service provides a new model of care and enables patients to leave hospital as soon as they are clinically stable and complete the remainder of their hospital care in the comfort of their own home. As a result, researchers are currently investigating about novel approaches that can enable the application to the HAH service management methodologies and tools originating from other industrial fields.

In particular, the management decisions can be hierarchically organized at the tactical and operational level. The HAH management at the tactical level deals with medium time decisions concerning the dimensioning and allocating resources such as doctors, nurses, medical facilities; acquiring authorizations to deliver care for certain pathologies; planning the routing for nurses and doctors [3], [7], [10], [17]. The HAH management problems at the operational level consists of real time decisions such as monitoring activities of daily living of inhabitants, monitoring the patients pathologies, and detecting the emergency events.

*This work was supported by the Italian Project PON RES NOVAE (Reti, Edifici, Strade, Nuovi Obiettivi Virtuosi per l'Ambiente e l'Energia).

${ }^{1}$ Maria Pia Fanti is with Electrical and Information Engineering Department, Politecnico di Bari, 70125 Bari, Italy mariapia.fanti@poliba.it

${ }^{2}$ Agostino Marcello Mangini is with Electrical and Information Engineering Department, Politecnico di Bari, 70125 Bari, Italy agostinomarcello.manginiepoliba.it

${ }^{3}$ Walter Ukovich is with Engineering and Architecture, University of Trieste, Trieste, Italy ukovich@units. it

${ }^{4}$ Jean-Jacques Lesage is with Ecole Normale Supérieure de Cachan, Cachan, France lesagedlurpa.ens-cachan. fr

${ }^{5}$ Kevin Viard is with Ecole Normale Supérieure de Cachan, Cachan, France kviardeens-cachan.fr
This paper specifies and models an Integrated System (IS) devoted to the HAH management at the operational level. To this aim we consider a smart home, i.e., a home equipped with sensors, actuators and communication networks connecting the electrical devices and allowing them to be remotely monitored and controlled. The IS is designed to monitor the daily living of an inhabitant into his dwelling, detect the possible troubles and accidents, and communicate with family, doctors and emergency services.

The research in the field of HAH systems deals with the following problems. Some contributions investigate about new sensors to be installed in smart homes for monitoring the wellness and the health of the inhabitants [8], [9], [12], [13] or for the suitable use and analysis of the sensor outputs [15]. Other studies consider the problem of the online location tracking based on a state estimator [5], [6]. Few contributions can be found about the integration of the sensor outputs and the system managing messages and alarms. In this context, Basilakis et al. [2] implement a decision support framework to analyze clinical information generated from subjects at their place of residence (home, residential care settings) and from other clinical environments. However, their work focuses on the efficient review and risk stratification of multiple patient records but does not assess the real time management and control of the inhabitants.

The aim of the presented IS is twofold: i) to monitor the inhabitant life and detecting inactivities and accidents; ii) to evaluate the gravity of the accident or the help request and sending the alarm messages. The IS is designed in a modular structure and each module is devoted to detect a particular diseases or accident. Moreover, each module is modelled in a Timed Petri Net (TPN) framework. In particular, a PN model offers several significant advantages with respect to the other discrete event system models: the graphical feature enables an effective modular modelling approach and the mathematical aspects efficiently allow the model verification and simulation.

The paper is structured as follows. Section II describes the architecture of the IS and Section III presents the TPN models of the modules composing the IS. Section IV draws the conclusion and the future research.

\section{THE INTEGRATED SYSTEM ARCHITECTURE}

This section presents the architecture and the activities of the IS that is responsible for the real time decisions of the HAH system at the operational level. In particular, 
Fig. 1 concisely describes the interactions among the IS, the involved actors and the services.

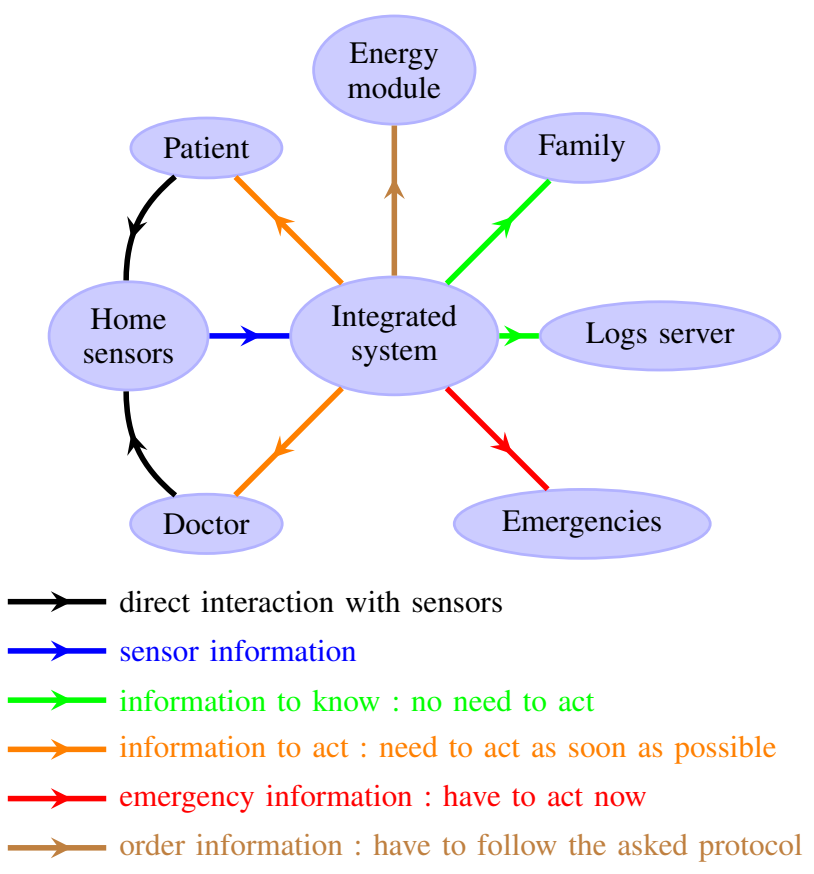

Fig. 1: Interactions of the Integrated System

More precisely, the IS receives information from the smart home sensors, analyses the messages and, on the basis of the detected events, it sends the messages to the family, the doctors or the emergency services.

The main emergency protocols that the IS can perform on the basis of the detected events concern: $i$ ) fall; ii) inactivities; iii) heart attacks and respiration troubles; $i v$ ) patient is out.

On the other hand, the operations that the IS can execute are the following:

- monitoring;

- sending alarms;

- trying to interact with the patient;

- calling emergencies;

- contacting doctor and family;

- enabling contact between call center and patient when he asks for help manually;

- interacting with the energy module that manages the home electric system of the patient.

The IS uses three types of messages on the basis of the alert priority: $i$ ) low level of alert, the messages are sent to the family; ii) medium level of alert, the messages are sent to the doctor; iii) high level of alert, the messages are sent to the emergency services.

Moreover, the IS interacts with the energy module sending commands to the electric devices, the heating, ventilation and air conditioning systems and the actions of the IS are stored in a $\log$ server.

\section{A. The Integrated System Structure}

Figure 2 shows the structure and the main components of the IS, i.e., the decision and the detection components.

The decision component manages and coordinates the reactions in the different cases of the inhabitant accidents and diseases. This component can be split in $m$ modules: each module is devoted to monitor and manage the decisions about a particular health attack or trouble. Here, we assume that the decision component is constituted of $m=4$ modules: heart attack and respiration trouble module (Module 1), fall event module (Module 2), inactivity event module (Module 3 ) and inhabitant goes out module (Module 4). Each module receives the messages about the detected events from the connected detection component and starts the suitable alarm and emergency protocols. To this aim, the decision component communicates with families, doctors and emergency systems.

Each module of the decision component is modelled in a Timed Petri Net (TPN) framework. Appendix A recalls some basic definitions of PNs: for additional details the interested reader can refer to [4] and [14].

Furthermore, the detection component monitors and detects the accidents and diseases of the inhabitants. Also such a component can be split in $n$ different detection modules. Each module is modelled by a Detection PN that is connected with a set of suitable sensors. The modules of the detection component have a dual role: i) receiving data from the sensors and converting the sensor outputs in events that can be symptoms of health problems of inhabitants; ii) sending the detected events to the decision components. Indeed, multiple sensors can be used to detect an event: for instance, cameras, movement sensors and floor sensors in a room can be employed to detect activity or inactivity, accidents and falls. Note that the modularity of the IS structure allows adding new sensors, detection and alarm procedures.

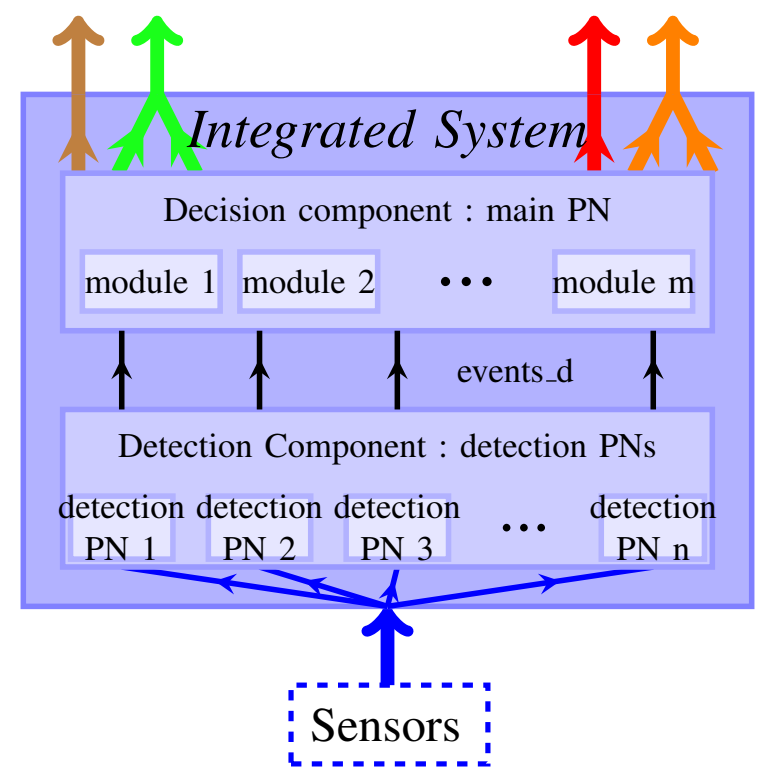

Fig. 2: Integrated System structure 


\section{THE INTEGRATED SYSTEM MODEL}

In this section, a case study is proposed in order to illustrate the IS model in the TPN framework.

\section{A. The Case Study}

We consider as a case study a smart home having an average size and planned for one person living inside. The topology of the apartment is organized as follows : the dining room constitutes an open space with the kitchen and the living room, one bedroom, one bathroom, a closet and a vestibule.

Every room is equipped with floor vibration sensors [1], [11], [16], giving three possible detected outputs : "small vibrations", "big vibrations" and "no vibration".

One or more interface boxes are installed on the wall including two buttons and an inter-phone. We denote the two buttons by: "ok_btn" and "alert", respectively. The interphone is used to establish a link between the patient and the call center when the patient pushes the "alert" button.

A special sensor on the room is connected to the phone line and the doctor can communicate that inhabitant is "ok".

A bed sensor [9], [12], [13] is in the bedroom, giving the cardiac pulse and respiration rhythm of the patient and a bathtub sensor [8], [12] is in the bathroom, giving the cardiac pulse of the patient.

\section{B. The Decision Component Model}

In this section we describe the TPNs modelling the four modules of the decision component for the considered case study. In each module some transitions are synchronized with a detection PN as it is shown in Fig. 3.

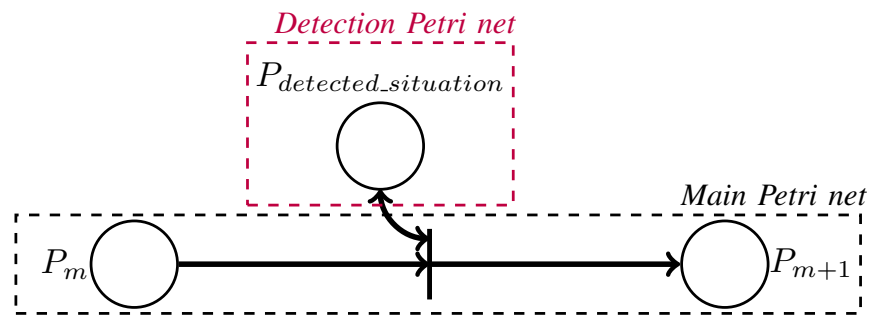

Fig. 3: Transition waiting for a detected_situation

A place belonging to a detection PN (i.e. the $P_{\text {detected_situation }}$ in Fig. 3) is in input of a transition named detected_situation_d of the module PN. When such a place is marked, transition detected_situation_d fires. For the sake of simplicity, we do not show the $P_{\text {detected_situation }}$ place in the PN module.

Each module includes place $P_{0}$ that, if it is marked, represents the situation "Everything is al-right": every output transition of this place needs a place of kind $P_{\text {detected_situation }}$ of the detection component. Note that in order to clearly show the meaning of transitions and places, we denote them by labels that explain their meaning.

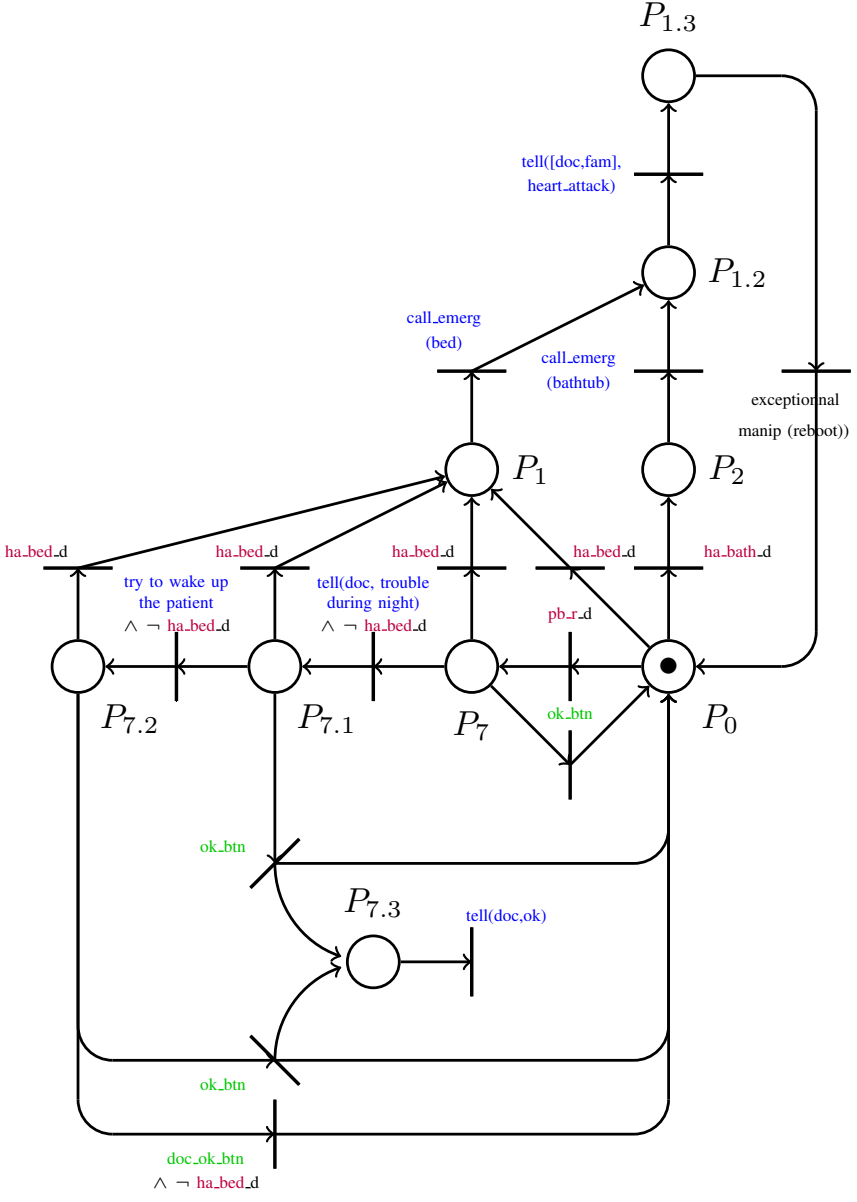

Fig. 4: PN 1 modelling Module 1: heart attack and respiration trouble.

The PN 1 shown in Fig. 4 models Module 1 that manages the events of heart attack or respiration troubles. In particular, places $P_{1}, P_{2}$ model the main detection conditions of PN 1 .

Place $P_{1}$ marked represents the occurrence of the "heart attack in bed". The input transitions of $P_{1}$ called "ha_bed_d" fire when the detection component detects an heart attack in the bed. In particular, the heart attack in bed can be detected immediately (transition "ha_bed_d" starting from $P_{0}$ ) or after some respiration troubles (transitions starting from $P_{7}$ ). In this last condition, if the heart attack is not detected and the inhabitant does not push the "ok" botton to indicate that he is fine, then the doctor is advised ( $P_{7.1}$ is marked). Three cases are now possible: 1$)$ the heart attack is detected; 2) the inhabitant pushes the "ok" botton (ok_btn fires and consequently $P_{0}$ and $P_{7.3}$ are marked); 3) the call center tries to wake up the inhabitant $P_{7.2}$. In particular, in case 2) the doctor is advised that the emergency is terminated (tell(doc,ok)).

Moreover, place $P_{2}$ marked initializes the "heart attack in bathtub" protocol. The input transition of $P_{2}$ is "ha_bath_d": this transition fires if an heart attack in the bathtub is detected by the detection component. Places $P_{1}$ and $P_{2}$ have only one 
output immediate transition : "call_emerg(bath)" that calls emergencies since an heart attack is detected in the patient's bathtub. Subsequently, the doctor and the family are alerted (tell([doc,fam],heart_attack)).

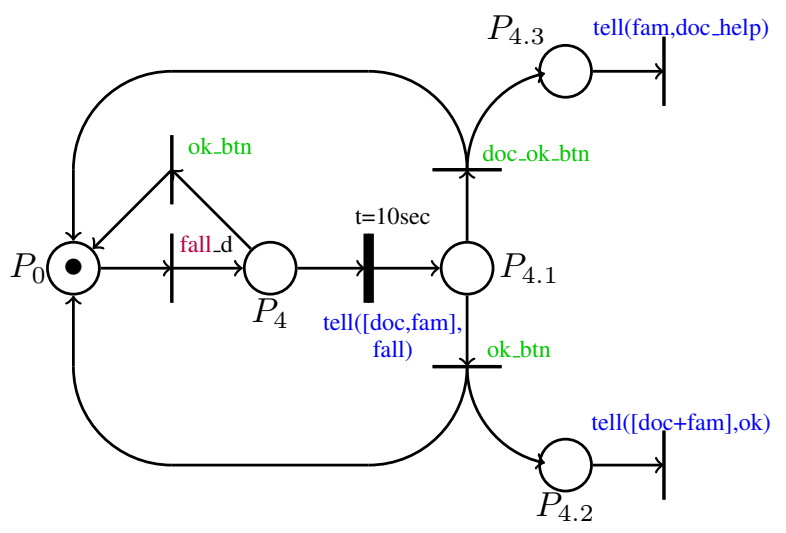

Fig. 5: PN 2 modelling Module 2: fall event.

Module 2 manages the fall accident and is modelled by PN 2 of Fig. 5. The marked place $P_{4}$ initializes the "fall" protocol. The description of input transition of $P_{4}$ is "fall_d": this means that the detection component has just detected a fall in the apartment. Place $P_{4}$ has two output transitions: "ok_btn" transition fires when the inhabitant pushes the "ok_btn" button to indicate that he is fine; the "tell([doc,fam],fall)" transition fires after a delay of 10 seconds and initializes the emergency procedure determined by a fall and the IS calls the doctor and family.

However, during the emergency procedure (place $P_{4.1}$ is marked), two transitions can eventually fire: transition "doc_ok_btn" fires if an ok comes from the doctor and transition "ok_btn" fires if the patient pushes the "ok_btn". In these cases, the IS sends a message of "no problem" to the family or to the doctor and the family, respectively (see transitions named "tell(fam, doc_ help)" and "tell([doc+fam],ok)").

Module 3 manages the reaction in case of inactivity and is modelled by the PN 3 of Fig. 6. Place $P_{6}$ is marked if the inactivity is detected and transition named "inact_d" fires: this means that the detection component considers that there is no movement in a room from long time. Place $P_{6}$ has the output immediate transition "tell([patient],inact)": the protocol for the inactivity starts. Place $P_{6.1}$ marked means that the IS is waiting for 5 minutes: if a "not inactivity" is received then transition "not_inact_d" fires; on the contrary the IS sends emergency messages to doctor and family since an inactivity is detected.

During the emergency procedure (place $P_{6.2}$ is marked), two transitions can fire: transition "doc_ok_btn" fires if an ok comes from the doctor and transition "not_inact_d" fires if a movement is detected in the room. In these cases, the IS sends a message of "no problem" to the family or to the doctor, the patient and the family, respectively (see transitions named "tell(fam, doc "tell([doc,pat,fam,emergencies],not inact)").

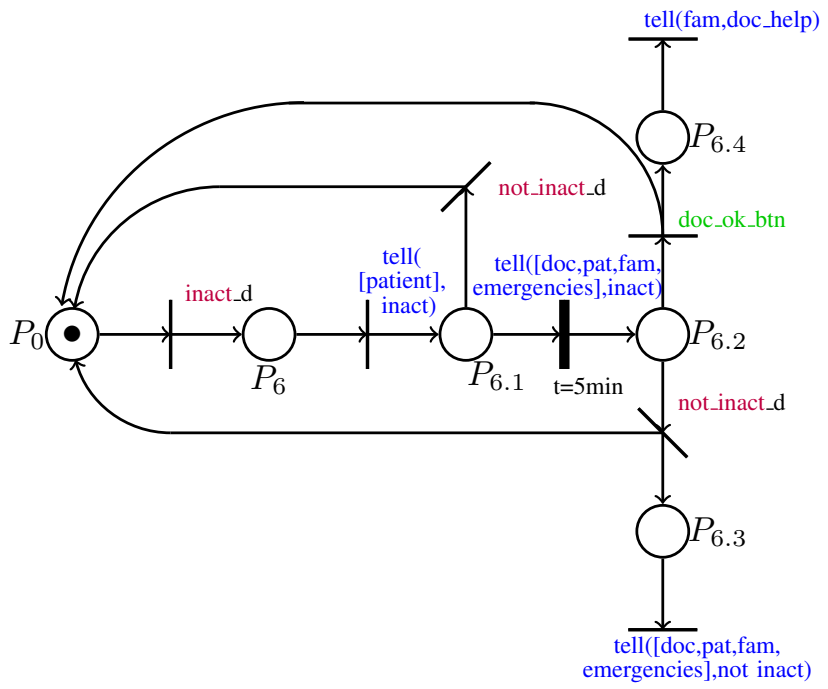

Fig. 6: PN 3 modelling Module 3: inactivity event.

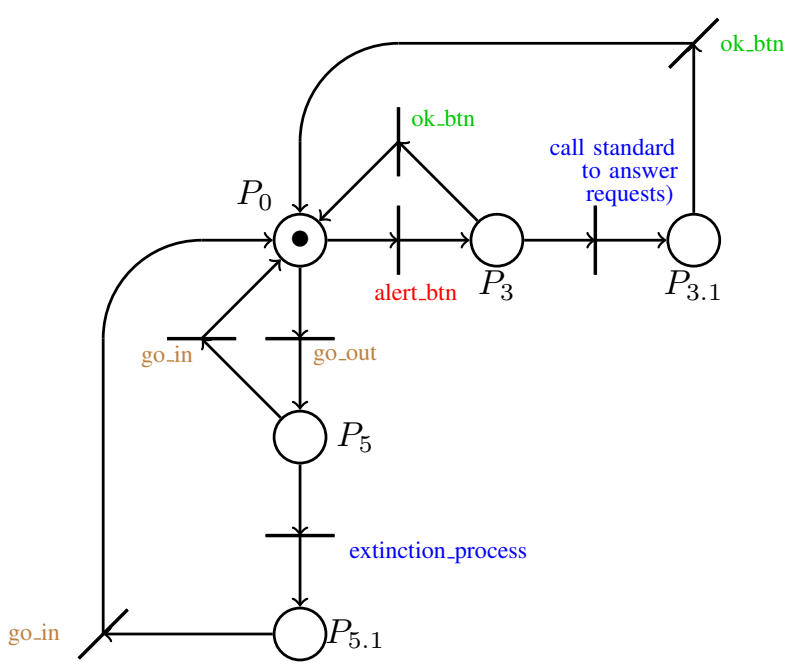

Fig. 7: PN 4 modelling the inhabitant goes out.

Module 4 manages the actions in case of alert given manually or the event "inhabitant goes out" and is modelled by PN 4 of Fig. 7. Place $P_{3}$ marked represents the initialisation of the "manual help asked" protocol. The input transition of $P_{3}$ is labelled "alert_btn" and it fires if the inhabitant pushes the alert button to ask help. The IS calls a doctor and put him in relation with the inhabitant.

On the other hand, place $P_{5}$ is marked if the input transition "go_out" fires: this means that the sensor of the home door detects that the inhabitant is out of the house. As a consequence, the IS starts a procedure to switch off the lights, the TV, the gas, some sensors, etc.

Place $P_{0}$ is again marked if transition "go_in" fires: the inhabitant goes back at home.

\section{The Detection Component Model}

In this subsection we describe the models of the detection component of the IS. In particular, we consider here three 
modules that are connected to floor sensors, bed sensors and bathtub sensors, respectively. Each module is described by a TPN.

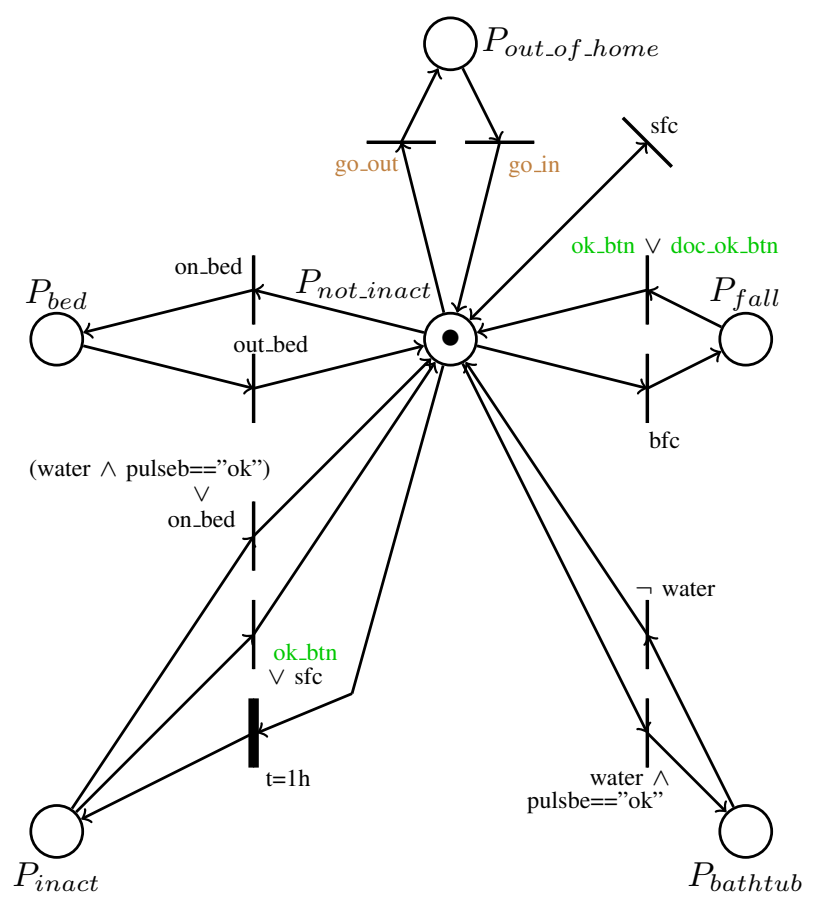

Fig. 8: Detection Petri net connected with the floor vibrations

The PN shown in Fig. 8 describes the module that detects the floor vibrations. It exhibits three main places that are connected with the floor sensors: $P_{\text {not_inact }}, P_{\text {inact }}$ and $P_{\text {fall }}$. Place $P_{\text {not_inact }}$ is marked if the inhabitant is not inactive. Starting from this state the following events may occur:

- the inhabitant goes to bed, i.e., a small vibration is detected (transition "on_bed" fires);

- the inhabitant goes out of home, i.e. a small vibration is detected (transition "go_out" fires);

- the inhabitant goes in the bathroom, i.e., a small vibration is detected (transition "water" fires);

- the inhabitant falls, i.e., a big vibration is detected (transition "bfc" fires);

- the inhabitant is inactive for one hour. In such a case place $P_{\text {inact }}$ becomes marked.

Moreover, in any case the inhabitant may use the button "OK" in order to declare that there is no problem and to block the emergency procedures.

Moreover, Fig. 9 shows the PN modelling the module connected with the bed sensors. It exhibits two places that are read by the decision component: $P_{p b_{-} r}$ and $P_{h a \_b e d}$.

In particular, place $P_{p b_{-} r}$ marked means that the IS detects that the patient has "respiration troubles in his bed". This situation is determined by a timed transition with a time delay of $10 \mathrm{sec}$. Also in this case the alarm situation can be cancelled if the patient or the doctors press the "ok_btn" (transition "ok_btn" or "doc_ok_btn" fire).

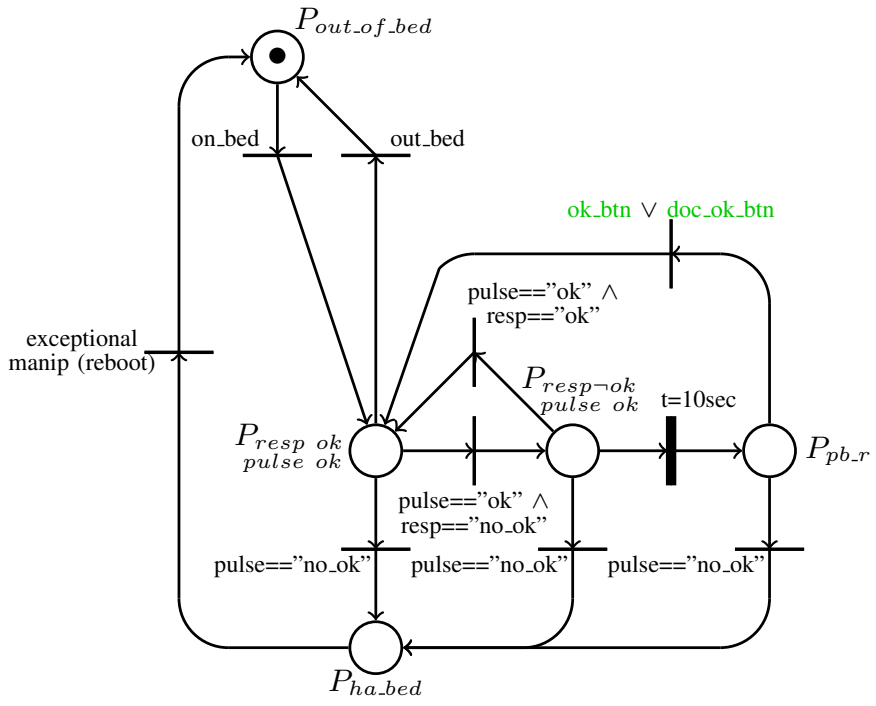

Fig. 9: Detection PN connected with the bed sensors.

Moreover, if place $P_{h a \_b e d}$ is marked means that "heart attack in bed" occurs. In this case a particular procedure is activated (transition exceptional manip (reboot) fires) and declares the patient out. All the system will work normally after the patient goes back to home.

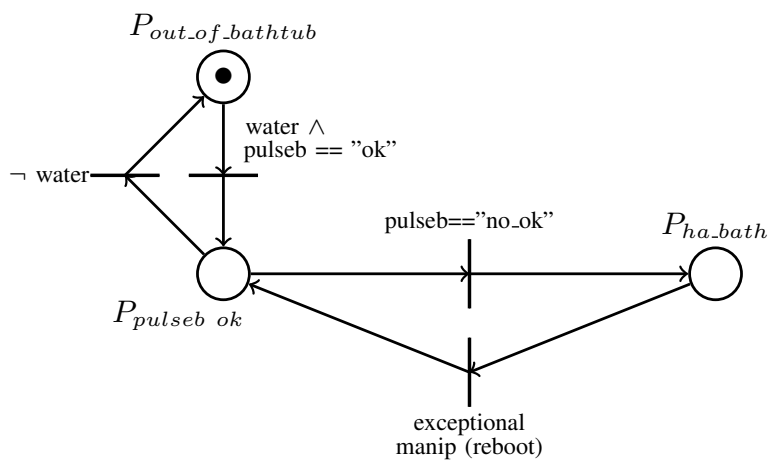

Fig. 10: Detection PN connected with the bathtub sensors

In addition, Fig. 10 shows the PN that detects the bathtub sensors: it exhibits one main place named $P_{h a-b a t h}$ that is marked if the pulse sensor detects a problem (transition pulseb $==$ "no_ok" fires). In such a case, an exceptional procedure starts (transition exceptional manip (reboot) fires) since the patient goes out. All the system will work normally when the patient goes back to home.

Moreover, place $P_{\text {pulseb ok }}$ marked symbolises that the patient is in the bathtub and $P_{\text {out_of_bathtub means that he }}$ is not on the bathtub. We consider that patient is come on the bathtub if there is water in front of the electrocardiogram integrated sensor and if a cardiac pulse is detected (water $\wedge$ pulseb $==$ "ok"). The patient is considered out of the bathtub if no water in front of electrocardiogram sensors is detected ( $\neg$ water). 


\section{Simulation}

The decision and detection component model, represented by the PNs and defined earlier, can be directly used to simulate the behavior of the IS. Discrete event simulations are used. In particular, sensor outputs are generated following probability distributions. In this way a set of events is created that fires the connected transitions. All transitions are fired as soon as possible. Moreover, the detection PNs are synchronized with the PNs modelling the modules of the decision component.

Before, we check by numerous simulations that each described PN is live, bounded and reversible [14]. After that, following the IS structure we assembly the different $\mathrm{PNs}$ in order to obtain the overall PN model. Moreover, a simulation has been realized by the freeware CPN Tools (http://cpntools.org/) to observe the behavior of the IS. In particular, one million of sensor outputs are generated during the simulation. The simulation allows checking the emergency protocols are properly performed and the properties of liveness, boundedness and reversibility are preserved.

\section{CONCLUSIONS}

This paper presents the architecture of an Integrated System (IS) devoted to manage at the operational level the Health-care At Home. To this aim, considering a smart home, the IS is designed to monitor the daily living of an inhabitant into his dwelling, detect the possible troubles and accidents, communicate with family, doctors and emergency services. The structure of the IS is described and its components are modelled in a Timed Petri Net framework. The structure modularity guaranties the generality of the system that can include additional detection and control modules without modifying its architecture.

Future works will integrate in the IS the telecare information and the patient records.

\section{APPENDIX}

\section{A. Definitions of Timed Petri Nets}

A TPN is a bipartite digraph described by the fivetuple $T P N=(P, T$, Pre, Post, $F)$, where $P$ is a set of places with $m$ elements, $T$ is a set of transitions with $n$ elements, matrices Pre : $P \times T \rightarrow \mathbb{N}^{m \times n}$ and Post : $P \times T \rightarrow \mathbb{N}^{m \times n}$ are the pre- and post-incidence matrices, respectively, that specify the arcs connecting places and transitions. More precisely, for each $P_{i} \in P$ and $T_{j} \in T$ element $\operatorname{Pre}\left(P_{i}, T_{j}\right)\left(\operatorname{Post}\left(P_{i}, T_{j}\right)\right)$ is equal to a natural number indicating the arc multiplicity if an arc going from $P_{i}$ to $T_{j}$ (from $T_{j}$ to $P_{i}$ ) exists, and it equals 0 otherwise. Moreover, function $F: T \rightarrow \mathbb{R}_{+}$specifies for each timed transition $T_{j} \in T$ the deterministic firing delay. Moreover, if it holds $F\left(T_{j}\right)=0$ then $T_{j}$ is an immediate transition.

The state of a TPN is given by its current marking, which is a mapping $M: P \rightarrow \mathbb{N}^{m}$, assigning to each place of the net a non-negative number of tokens. A TPN system $\left\langle T P N, M_{0}\right\rangle$ is a TPN with initial marking $M_{0}$. In addition, a transition $T_{j} \in T$ is enabled at a marking $M$ if and only if for each $P_{i} \in P$, it holds: $M\left(P_{i}\right) \geq \operatorname{Pre}\left(P_{i}, T_{j}\right)$. When $T_{j}$ fires, the new marking is computed by the PN state equation $M^{\prime}=M-\operatorname{Pre}\left(., T_{j}\right)+\operatorname{Post}\left(., T_{j}\right)$.

\section{REFERENCES}

[1] M.Alwan, P.J. Rajendran, S. Kell, D. Mack, S. Dalal, M. Wolfe, R. Felder (2006). A Smart and Passive Floor-Vibration Based Fall Detector for Elderly. In the Proceedings of the Information and Communication Technologies: From Theory to Applications (ICTTA'06), Vol. 2, Damascus, pp. 1003-1007.

[2] J. Basilakis, N.H. Lovell, B.G. Celler (2007). A Decision Support Architecture for Telecare Patient Management of Chronic and Complex Disease. In the Proceedings of the 29th Annual International Conference on IEEE Engineering in Medicine and Biology Society (EMBS), Lyon, France, August 22-26, pp. 4335-4338.

[3] M. Blais, S.D. Lapierre, G. Lapierre (2003). Solving a home-care districting problem in an urban setting. Journal of the Operation Research Society, Vol. 54, No. 11, pp. 1141-1147.

[4] C.G. Cassandras and S. Lafortune (2008). Introduction to Discrete Event Systems. Springer, Second Edition, New York, NY, USA.

[5] M. Danancher, J.J. Lesage, L. Litz, G. Faraut (2013). Online Location Tracking of a Single Inhabitant based on a State Estimator. In the Proceedings of the IEEE International Conference on Systems, Man, and Cybernetics - SMC 2013, Manchester, United Kingdom, October 13-16.

[6] M. Danancher, J.J. Lesage, L. Litz, G. Faraut (2013). A Discrete Event Model for Multiple Inhabitants Location Tracking. In the Proceedings of the IEEE International Conference on Automation Science and Engineering (CASE), Madison, WI, USA, August 17-20, pp. 910-915.

[7] V. De Angelis (1998). Planning home assistance for AIDS patients in the city of Rome, Italy. Interfaces, Vol. 28, No. 3, pp. 75-83.

[8] Y. Fujital, K. Watanabe, K. Kobayashi, Y. Kurihara (2011). Development of System for Unrestrained Measurement of Vital Signs in the Bathroom. In the Proceedings of the SICE Annual Conference,Tokyo, September 13-18, pp. 2322-2325.

[9] L. Jiang, Z. Tang, Z. Liu, W. Chen, K.-I. Kitamura, T. Nemoto (2012). Automatic Sleep Monitoring System for Home Healthcare. In the Proceedings of the IEEE-EMBS International Conference on Biomedical and Health Informatics (BHI 2012), Hong Kong, Jan. 5-7, pp. 894-897.

[10] E. Lanzarone, A. Matta, E. Sahin (2012). Operations Management Applied to Home Care Services: The Problem of Assigning Human Resources to Patients. IEEE Transactions on System, Man, and Cybernetics-Part A: Systems and Humans, Vol. 42, No. 6, pp. 13461362.

[11] D. Litvak, I. Gannot, Y. Zigel (2008). Fall Detection of Elderly through Floor Vibrations and Sound. In the Proceedings of the 30th Annual International Conference on IEEE Engineering in Medicine and Biology Society (EMBS), Vancouver, BC, August 20-25, pp. 4632-4635.

[12] K. Motoi, M. Ogawa, H. Ueno, Y. Kuwae, A. Ikarashi, T. Yuji, Y. Higashi, S. Tanaka, T. Fujimoto, H. Asanoi, K.-I. Yamakoshi (2009). A Fully Automated Health-Care Monitoring at Home Without Attachment of Any Biological Sensors and its Clinical Evaluation. In the Proceedings of the 31st Annual International Conference on IEEE Engineering in Medicine and Biology Society (EMBS), Minneapolis, MN, September 3-6, pp. 4323-4326.

[13] S. Nukaya, T. Shino, Y. Kurihara, K. Watanabe, H. Tanaka (2012). Noninvasive Bed Sensing of Human Biosignals Via Piezoceramic Devices Sandwiched Between the Floor and Bed. IEEE Sensors journal, Vol. 12, No. 3, pp. 431-438.

[14] J.L. Peterson (1981). Petri Net Theory and the Modelling of System. Prentice Hall, Englewood Cliffs, NJ, USA.

[15] T.B.T. Truong, F. Frizon de Lamotte, J.Ph. Diguet, F. Said-Hocine (2010). Alert Management for Home Healthcare Based on Home Automation Analysis. In the Proceedings of the 52nd Annual International Conference of the IEEE Engineering in Medicine and Biology Society (EMBS), Buenos Aires, Argentina, August 31-September 4.

[16] F. Werner, J. Diermaier, S. Schmid, P. Panek (2011). Fall Detection with Distributed Floor-mounted Accelerometers. In the Proceedings of the 5th International Conference on Pervasive Computing Technologies for Healthcare (PervasiveHealth), Dublin, May 23-26, pp. 354-361.

[17] H. Zhou, M. Hou, C.Wang, M.C. Zhou (2012). An Efficient Outpatient Scheduling Approach. IEEE Transactions on Automation Science and Engineering, Vol. 9, No. 4, pp. 701-709. 